\title{
Cuttings Transport Behavior and Simulation Analysis Affected by Hole Cleaning Tool
}

\author{
Sun Xiaofeng*, Yan Tie, Shao Shuai, Wang Kelin and Luan Shizhu \\ College of Petroleum Engineering, Northeast Petroleum University, Daqing, Heilongjiang, China
}

\begin{abstract}
Researchers have designed many kinds of hole cleaning tools to improve hole cleaning condition in the complex structural wells. In this paper a mathematical model was built to analyzing the cutting particles movement on the helical blades of hole cleaning tool. The relationships between blade helical angles and cuttings transport velocity in tangential direction and axial direction are obtained by solving the model. The results show that blade helical angle as a controlling variable has a significant impact on application effect of the spiral hole cleaning tool; tangential cuttings velocity increases and axial cuttings velocity decreases with the increase in the helical angle and adjusting the helical angle can reach the required cuttings velocity.
\end{abstract}

Keywords: Hole cleaning, hole cleaning tool, helical angle, cuttings bed, complex structural well.

\section{INTRODUCTION}

Field experiences $[1,2]$ have shown that cuttings bed is always formed while drilling horizontal and ERW wells, which leads to high drag and torque, pipe sticking, slower rate of penetration, formation fractures and well bore steering problems [3], and also affects the subsequent jobs such as cementing and casing problems. Therefore, it is very important for horizontal and ERW wells to enhance cuttings transport efficiency. Many researchers have developed a series of helical hole cleaning tools to improve hole cleaning condition and obtained good results [4-6]. These tools have helical blades on their surface, and outer diameters of these blades are close to drill pipe sub. The helical tool is connected with drill pipe so that it can make full use of the drill pipe rotation. Its working mechanism is that with helical tool rotation a special flow field is formed in annuli, and cuttings bed is destroyed and particles are accelerated. In recent years, most researches applied CFD software to study the effect of tool rotation on the flow behavior of drilling fluid to predict the cuttings transport affected by the tool $[7,8]$. This paper analyzed hole cleaning performance of the helical tool used with drill pipe. Movement equations were also developed for the particles on the blade, and numerical simulations were used to analyze the characteristics of particle movement. These researches can provide a reference for the similar tool design.

\section{FORCES AND MOTION EQUATIONS FOR PAR- TICLE ON HELICAL BLADES}

\subsection{The Motion Equation in Vertical Segment}

The basic structure and working diagram of hole cleaning tool in vertical segment are shown in Fig. (1) and Fig. (2). In

*Address correspondence to this author at the Daqing High-Tech Industrial Development Zone No.199, Daqing, Heilongjiang Province, 163318, China; Tel: 0459-6503521; Fax: 0459-6503482;

E-mails: suneye@126.com; 493688306@qq.com order to analyze the movement behavior of cuttings contacted with the blades, the following hypotheses are made within the range that will not deteriorate the model's accuracy: i) no interaction among cuttings, ii) spherical cuttings with the same diameter and without rotation, and iii) incompressible steady-state flow.

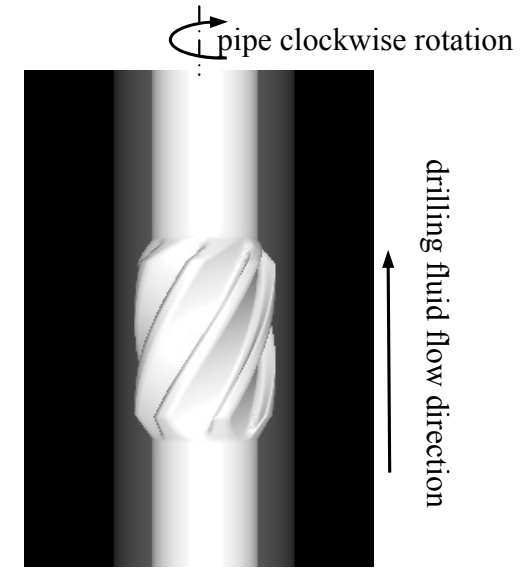

Fig. (1). The working diagram of hole cleaning tool in vertical segment.

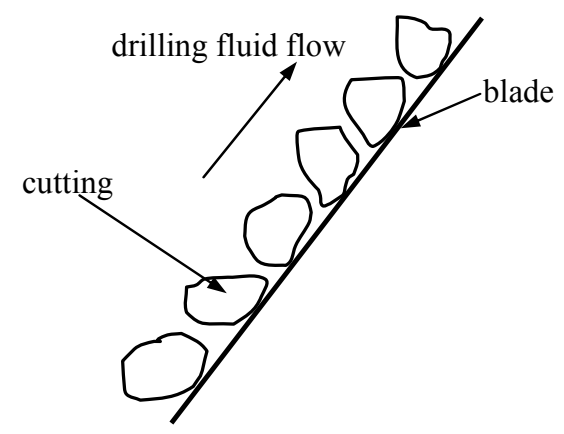

Fig. (2). Distribution of cuttings at the blade. 
As shown in Fig. (3), the movement behavior of the particle at Spiral curved surface is depicted in a certain annular fluid flow. When $t=0$, the particle is located in $\mathrm{M}_{0}$ point. As $t=t_{0}$, the cutting particle moves to $\mathrm{M}$ point, and $\mathrm{M}_{0}$ point at helicoid rotates to $\mathrm{M}_{0}{ }^{\prime}$ point. During the process of particle movement, $S_{\mathrm{a}}$ is the absolute transport trajectory, $V_{\mathrm{a}}$ is the absolute velocity, $S_{\mathrm{r}}$ is the relative transport trajectory, $V_{\mathrm{r}}$ is the relative velocity, and $V_{\mathrm{e}}$ is the transport velocity. The particle force analysis as follow:

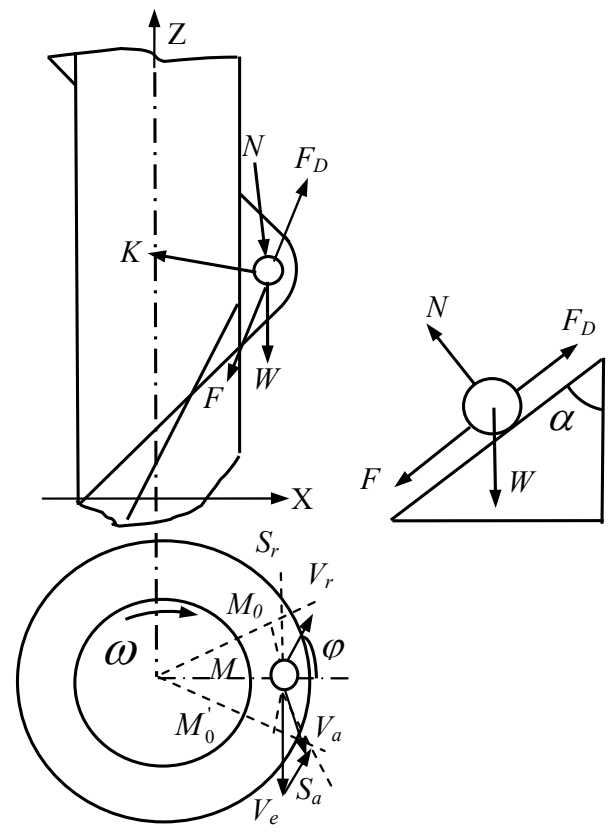

Fig. (3). Movement and forces for a cutting at Spiral curved surface.

Gravity force. Due to the buoyancy force acting on the particle in the fluid flow, the gravity force is expressed as:

$$
W=\frac{4}{3} \pi R^{3}\left(\rho_{s}-\rho\right) g
$$

Thrust force. The blade generates a blade thrust at the particle when drill pipe is rotating, and the force direction is perpendicular to the blade. The thrust force can be calculated as following equation:

$$
N=W \sin \alpha
$$

Frictional force. The frictional force between cutting and blade can be written as:.

$$
F=f N
$$

Drag force. The particle in the moving fluid experiences a drag force paralleling to the direction of upstream velocity. It is defined as:

$$
F_{D}=\frac{1}{2} C_{D} s \rho\left(V-u_{f}\right)\left|V-u_{f}\right|
$$

Centrifugal force. As rotating with the helicoid, there is a centrifugal force acting on cutting. It is defined as:

$$
D=m r \omega^{2} \ldots
$$

Inertia force. Due to the particle movement in the noninertial system, there is a Corioli inertial force acting on the particle, which can be written as following equation:

$$
K=2 m V_{r} \omega
$$

The relative motion of particle at helicoid is considered as the uniform linear motion, so the inertia force of relative motion is zero.

Among the above analyses, the tangential and axial velocity equations are derived based on the mechanical equilibrium.

$$
\begin{aligned}
& V_{r x}=\left(m r \omega^{2}+f N \sin \alpha \cos \varphi-F_{D} \sin \alpha \cos \varphi\right) /(2 m \omega \cos \alpha) \\
& V_{r z}=\left(W+f N \cos \alpha-F_{D} \cos \alpha\right) /(2 m \omega \sin \alpha)
\end{aligned}
$$

The absolute velocity of particle movement can be defined through the superposition theorem.

$$
\begin{aligned}
& V_{x}=\sqrt{\left(V_{r x}\right)^{2}+(2 \pi n r)^{2}-4 \pi n r\left|V_{r x}\right| \cos \left(\frac{\pi}{2}-\varphi\right)} \\
& V_{z}=V_{r z}+\frac{2 \partial n r}{\tan a ́}
\end{aligned}
$$

\subsection{The Movement Equation in Inclined Segment}

In inclined segment as shown in Fig. (4), the particles will be excavated by the tool and transported to the surface by the high fluid flow when hole cleaning tool contacts with the cuttings bed. Based on this theory of vertical segment, the inclination is introduced to depict the particle movement in inclined segment.

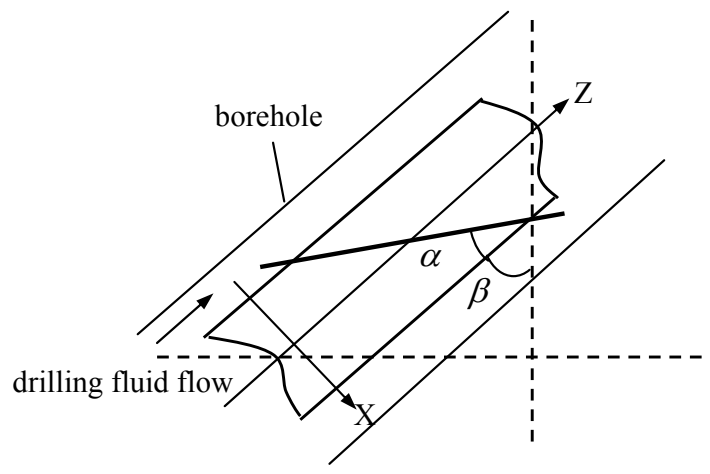

Fig. (4). The diagram of hole cleaning tool in inclined segment.

While hole cleaning tool works at the angle of $\beta$, the blade thrusts the particle with the force $(\mathrm{N})$, and the direction is perpendicular to the blade.

$$
N=W|\sin (\pi-\alpha-\beta)|
$$

Equation (12) and (13) are used to describe the tangential and axial velocity of cutting movement, respectively:

$$
\begin{aligned}
& V_{r x}=\left(m r \omega^{2}+W \sin \beta+f N \sin \alpha \cos \varphi-F_{D} \sin \alpha \cos \varphi\right) /(2 m \omega \cos \alpha) \\
& V_{r z}=\left(W \cos \beta+f N \cos \alpha-F_{D} \cos \alpha\right) /(2 m \omega \sin \alpha)
\end{aligned}
$$

In addition, the absolute velocity of cutting movement can be got through equations (9) and (10). 
3. CUTTINGS TRANSPORT NUMERICAL SIMULATION AND ANALYSIS

\subsection{Numerical Simulation Conditions}

The above mathematical models are used to simulate the cuttings transport behavior affected by hole cleaning tool in different working conditions, and the conditions as follow:

Fig. (5) shows the transverse of the tool. The blade radius is $85 \mathrm{~mm}$, transverse intersection angle is $50^{\circ}$, drilling fluid density is $1.2 \mathrm{~g} / \mathrm{cm}^{3}$, cutting density is $2.5 \mathrm{~g} / \mathrm{cm}^{3}$, cuttings radius is $0.2 \mathrm{~mm}$. The helical angle, pipe rotational speed, drilling fluid flow rate, inclination are listed in (Table 1).

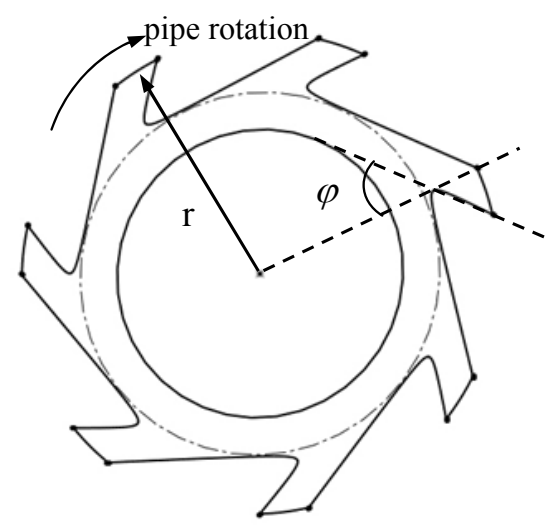

Fig. (5). The transverse section of the tool.
Table 1. Data Required by Simulations

\begin{tabular}{|c|c|}
\hline Helical angle $\left({ }^{\circ}\right)$ & $25 ; 30 ; 35 ; 40 ; 45 ; 50 ; 55 ; 60 ; 65 ; 70$ \\
\hline Drilling fluid flow rate $(\mathrm{m} / \mathrm{s})$ & $1.2 ; 1.5 ; 2$ \\
\hline Pipe rotational speed $(\mathrm{rpm})$ & $80 ; 100 ; 120$ \\
\hline Inclination $\left({ }^{\circ}\right)$ & $60 ; 70 ; 80$ \\
\hline
\end{tabular}

\subsection{Results and Discussion}

Cuttings bed is more difficult to be removed at the angle of $60^{\circ}[9,10]$. Therefore, the effect of the helical angle, pipe rotational speed and drilling fluid flow rate on the particle movement was simulated in the section at angle of $60^{\circ}$, and the results are shown in Fig. (6) to Fig. (9).

As shown in Fig. (6), with the increase at helical angle, tangential velocity of particle movement increases, but axial velocity decreases, and is close to zero when the helical angle towards to 70 degrees.

Fig. (7) indicates cuting particles were obviously accelerated by tool rotation with the increase of rotational speed. For a given drill pipe rotational speed, with the increase at helical angle resultant velocity of particle movement first decreases, then increases. In terms of energy, the tool rotation does positive work on particles so that the velocity of particles is increased.

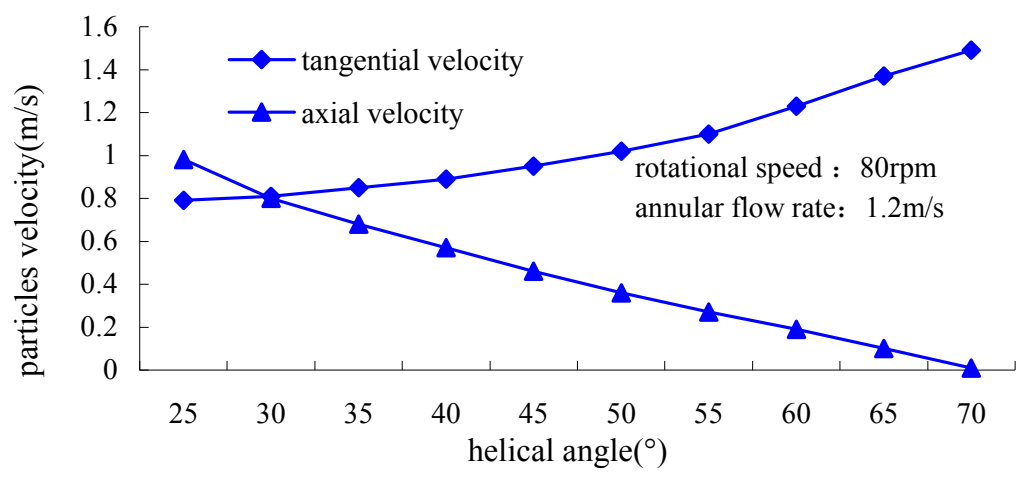

Fig. (6). Relationship between helical angle and cutting particles velocity.

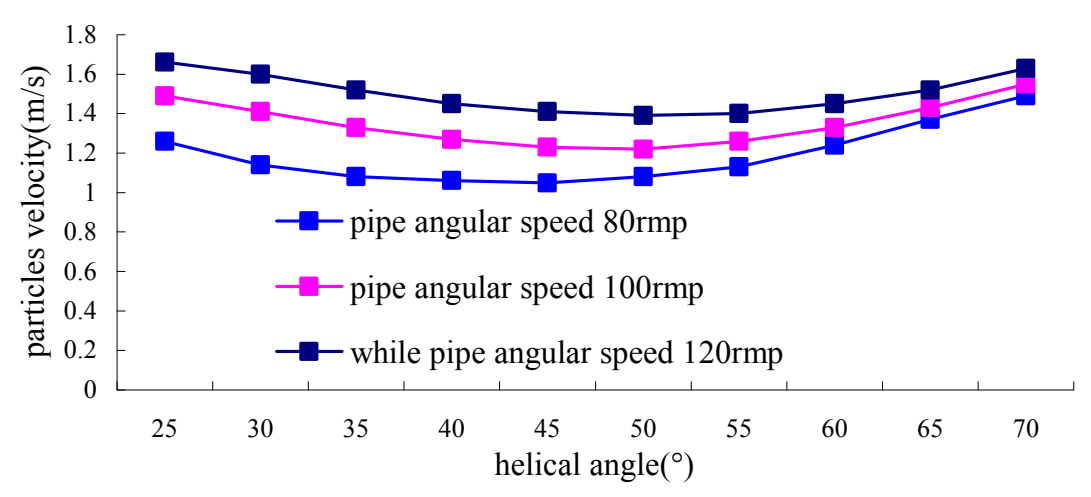

Fig. (7). Relationship between resultant velocity of cutting particles movement and helical angle with different pipe rotational speed at 1.2 $\mathrm{m} / \mathrm{s}$ annular flow rate. 
Fig. (8) and Fig. (9) show that increasing drilling fluid flow rate results in a higher tangential velocity and axial velocity of particle. For a given drilling fluid flow rate, tangential velocity increases and axial velocity decreases with the increase at helical angle. Therefore, the higher drilling fluid flow rates contribute to cuttings transport.

Fig. (10) indicates that the resultant velocity of particle movement gradually increases with the increase at inclination, especially, there is a large range at inclination from vertical to 60 degrees. In addition, a critical helical angle also is found, and it should be avoided in process of designing helical tool.

The comparison of the above figures shows the helical angle has an obvious effect on particle movement velocity. With the increase at the helical angle, tangential velocity of particle movement also increases. However, when the helical angle decreases, axial velocity of particle movement also increases. The cuttings are excavated from the cuttings bed by higher tangential velocity, and are transported to the surface by higher axial velocity.

\section{CONCLUSIONS}

(1) The helical tool used for hole cleaning can make cuttings accelerated obviously, and cutting particle velocity can approach the drilling fluid flow rate, which is helpful for cuttings transport and preventing the formation of cuttings bed again.

(2) The mathematical models in this paper can quantitatively describe cuttings transport behavior after cuttings are through hole cleaning tool, especially, the tangential velocity and axial velocity of cuttings are calculated, which can provide the theoretic basis for designing this type of tools.

(3) Helical angle of hole cleaning tool has a great effect on the particle movement velocity. When designing the tool, the helical angle should be gradually adjusted until the required particle velocity is obtained.

\section{CONFLICT OF INTEREST}

The author(s) confirm that this article content has no conflicts of interest.

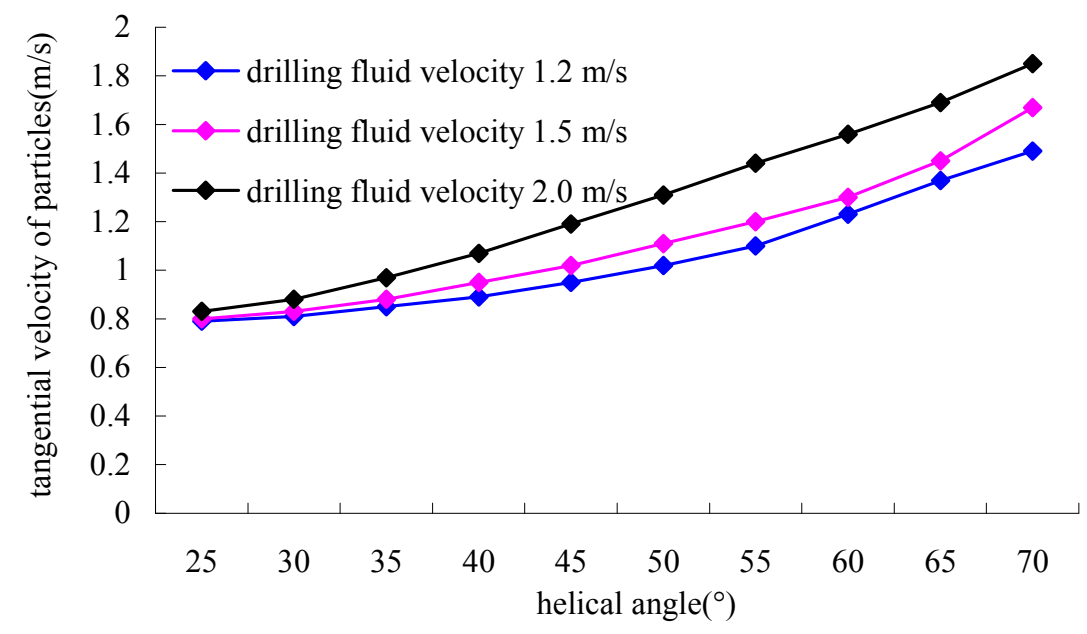

Fig. (8). Tangential velocity of cuttings with different annular fluid flow rate at 80rpm pipe rotational speed.

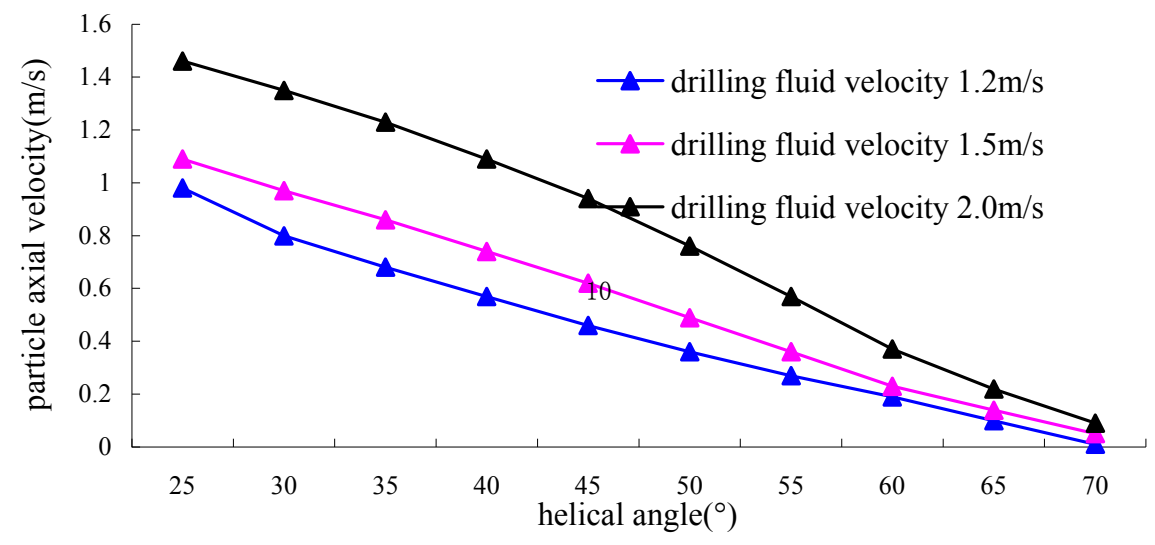

Fig. (9). Axial velocity of cuttings with different annular flow rate at $80 \mathrm{rpm}$ pipe rotational speed. 


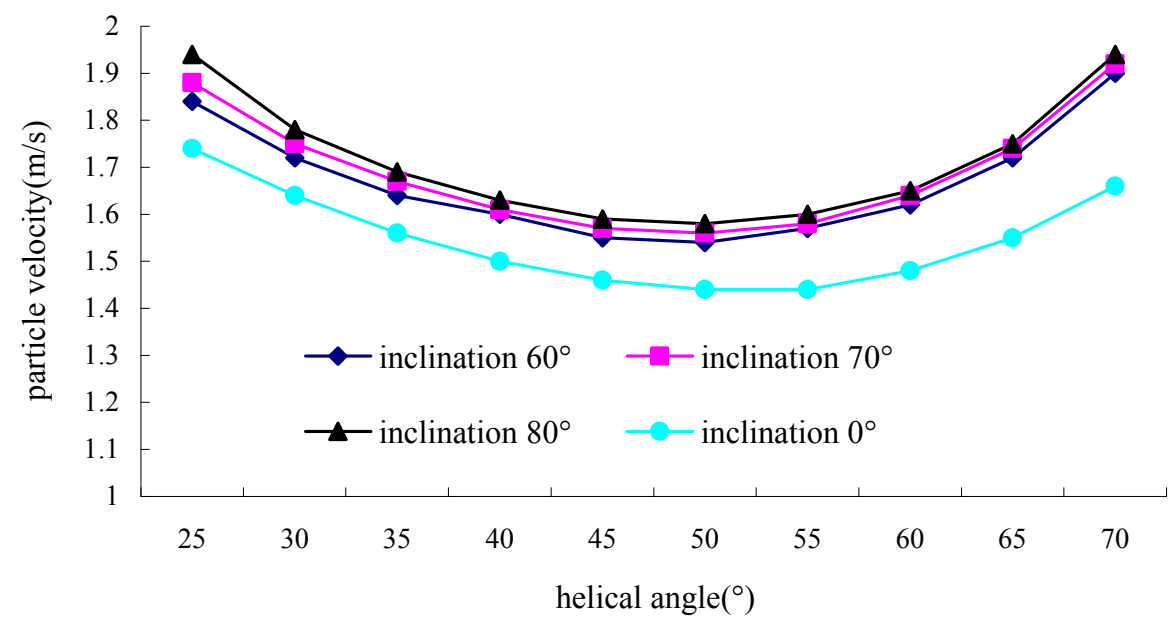

Fig. (10). Resultant velocity of cutting movement with different inclination at $2.0 \mathrm{~m} / \mathrm{s}$ annular flow rate and $100 \mathrm{rpm}$ pipe rotational speed.

\section{ACKNOWLEDGEMENT}

The support of National Science and Technology Major Project of China (No. 2011ZX05009-005) and the National Natural Science Funds of China (No. 51174043 and No. 51204056) are gratefully acknowledged.

\section{NOMENCLATURE}

$$
\begin{aligned}
& t=\text { time, } \mathrm{s} \\
& C_{D}=\text { drag coefficient, dimensionless } \\
& \rho_{s}=\text { cutting density, } \mathrm{kg} / \mathrm{m}^{3} \\
& \rho=\text { drilling fluid density, } \mathrm{kg} / \mathrm{m}^{3} \\
& R=\text { cutting particle radius, } \mathrm{m} \\
& r=\text { blade radius, } \mathrm{m} \\
& \alpha=\text { helical angle, }^{\circ} \\
& f \quad=\quad \text { friction coefficient } \\
& m=\text { particle mass, } \mathrm{kg} \\
& n=\text { rotational speed of pipe, } \mathrm{rpm} \\
& \varphi=\text { transverse intersection angle, }^{\circ} \\
& s=\text { spherical great circle area, } \mathrm{m}^{2} \\
& \omega=\text { pipe angular speed, } \mathrm{rad} / \mathrm{s} \\
& u_{f}=\text { drilling fluid velocity, } \mathrm{m} / \mathrm{s} \\
& V \quad=\quad \text { cutting particle velocity, } \mathrm{m} / \mathrm{s} \\
& V_{r x}=\text { relative tangential velocity of particle, } \mathrm{m} / \mathrm{s} \\
& V_{r z}=\text { relative axial velocity of particle, } \mathrm{m} / \mathrm{s}
\end{aligned}
$$

$$
\begin{aligned}
& V_{x}=\text { tangential velocity of particle, } \mathrm{m} / \mathrm{s} \\
& V_{z}=\text { axial velocity of particle, } \mathrm{m} / \mathrm{s}
\end{aligned}
$$

\section{REFERENCES}

[1] Z.F. Wang. "Cuttings bed clearage of complex structural wells", Oil Drilling\& Production Technology, vol. 31, pp. 102-104, 2009.

[2] L.D. Guo. "Methods to solve cuttings bed of high-inclination and extended reach well in Jidong oilfield", Exploration Engineering, vol. 5, pp. 58-59, 2004.

[3] A.M. Kamp, and M. Rivero."Layer modelling for cuttings transport in highly inclined wellbores", In: Latin American and Caribbean Petroleum Engineering Conference, 1999, pp. 1-12.

[4] J.R. Hu. "New technology combining cuttings removal and drilling", Drilling \& Production Technology, vol. 24, pp. 5-6, 2001.

[5] M.Q. Duan, S. Miska, M.J. Yu, N. Takach, R. Ahmed, and C. Zettner. "Critical conditions for effective sand-sized- solids transport in horizontal and high-angle wells", Drilling \& Completion, vol. 24, pp. 229-238, 2007.

[6] Z.H. Wu, L.R. We, and Y.F. Nie. "Using rock bed dismantle tool and expandable casing holder to improve well bore quality", Oil Field Equipment, vol. 35, pp. 59-62, 2006.

[7] H.Y. Sun, "Design of cuttings bed remover and simulation of flow field created by it", China Petroleum Machinery, vol. 37, pp. 38$41,2009$.

[8] F. Chen, Q.F. Di, P.B. Yuan, W.C. Wang, J.L. Yao, Y.Q. Zhou, and G.X. Zhai. "Mechanism of an effective hydroclean drill pipe for hole cleaning", Acta Petrolei Sinica, vol. 33, pp. 298-303, 2012.

[9] M.H. Yang, Y. Huang, Y.H. Zhai, X.Y. Li, and W.G. Wang. "Research and application of annular cuttings bed thickness evaluation model for high angle wellbores including horizontal wells", Natural Gas Industry, vol. 28, pp. 62-64, 2008.

[10] Z.M. Wang, X.L. Guo, S.J. Zhang, and D.L. Gao, "Casing program design method for Liuhua mega-extended-reach wells in south China sea", Oil Drilling \& Production Technology, vol. 28, pp. 4-8, 2006.

Received: May 15, 2013

(C) Xiaofeng et al.; Licensee Bentham Open.

This is an open access article licensed under the terms of the Creative Commons Attribution Non-Commercial License (http://creativecommons.org/licenses/by-nc/3.0/) which permits unrestricted, non-commercial use, distribution and reproduction in any medium, provided the work is properly cited. 